\title{
CFD Modelling of Thermo-electric Devices for Thermal Management in Downhole Tools
}

Rohitha Weerasinghe ${ }^{1}$, Thomas P. Hughes ${ }^{1}$

${ }^{1}$ Faculty of Environment and Technology, University of the West of England, Coldharbour Lane, Bristol, BS16 1QY, UK

* Corresponding Author: Rohitha.weerasinghe@uwe.ac.uk, +44 1173287137

\section{Abstract}

Investigation of oil and gas fields using vertical seismic profiling subjects the electronics used to extreme temperatures and pressures. As wells become deeper, the need for effective thermal management in instrumentation and the demands placed on the tools increases. Various techniques including vacuum insulation, heat pipes, phase change media and thermo-electric cooling (TEC) devices are used to maximise the time equipment can be exposed to these hostile conditions. The performance of such devices is temperature dependent and the level of cooling attained is a function of thermal efficiency. This work examines the effect of different TEC devices on the thermal performance of a commercially available Vertical Seismic Profiling tool. Experimental validation of the model is based on measurements and is presented here. The predictions agree closely with the measurements and the model can be used as a computational tool reducing cost and time in measurements.

\section{Introduction}

Oil and gas are still of primary importance to future societies as we have not yet perfected the technology surrounding alternative fuels. Seismic surveys are a critical element of the search for oil and gas exploration. In a seismic survey, the propagation of elastic waves through the rock gives an indication of the sub surface distribution of different rock types and thus the probability of finding a viable source of hydrocarbons. The seismic source varies depending on the application but may be dynamite, an air gun, or a vibrating plate to produce waves at a range of frequencies. In a surface seismic study usually both the source and the receivers (Igeophones"; sensitive ground velocity sensors) are located at the surface and the reflected waves are analysed. In a Vertical Seismic Profiling borehole investigation the receivers are located within the borehole and the source is usually located at the surface or, less frequently, downhole. Borehole seismic data can provide calibrated, high resolution data that can be used alone, or in conjunction with surface seismic data in order to make exploration decisions and thus is a valuable technique for well characterisation. An additional application for borehole seismic logging tools is the monitoring of hydraulic fracturing (fracking) sites.

\subsection{Instrumentation used in downhole tools}

Instrumentation used in borehole seismic investigations needs to be extremely sensitive in order to capture the micro-seismic waves at the receiver and large volumes of data must be sent back to the surface-in the case of continuous monitoring surveys this must be in near real time. This process requires sensitive and sophisticated electronics to be exposed to extremely hostile environments; depending on the wellbeing surveyed this may be pressures of up to $30,000 \mathrm{psi}(2000 \mathrm{bar}$ or $206 \mathrm{MPa}$ ), temperatures of greater than $200 \mathrm{C}$ [3] and often in environments with high concentrations of $\mathrm{H}_{2} \mathrm{~S}$. In downhole measuring the time taken can be from a single day to a few months depending on application and location.

\subsection{The requirement of cooling}

Whilst electronics manufacturers are continually pushing to increase the service temperatures of their products, many devices are not able to survive at the temperatures encountered in a borehole and thus it is necessary to provide a degree of cooling to the electronics within downhole tools. Various strategies have been deployed to achieve this over the last 30 years including vacuum (Dewar) insulation, eutectic alloys which extend the time available downhole [1] and various active cooling technologies [2] Unlike in conventional electronics cooling applications, the need for the system to be hermetically sealed and withstand high pressures prevents the use of forced air cooling. In addition to shielding the electronics from the heat of the borehole fluid, a further challenge is in dissipating the heat produced by the electronics themselves. Various active cooling techniques have been evaluated in the literature. Many of these use refrigerant recirculation techniques which when employed in a "logging whilst drilling" scenario can be very effective but in a VSP application the noise generated by the system makes this impractical, or at the least exceedingly challenging to implement.

\section{Methodology}

The borehole tooling used in this study was supplied by Avalon Sciences Ltd that is being used in measuring. It comprises a steel pressure barrel which houses the geophones, a mechanism to operate an arm which clamps the tool to the wall of the borehole and a module containing the digital electronics which perform the signal processing function. These electronics are housed within a vacuum insulated vessel and active cooling is provided by a Thermoelectric Cooler (TEC) module. 


\subsection{The mathematical model}

The differential temperature across the thermoelectric device is due to the Seebeck effect. The temperature difference across the unit is proportional to the voltage drop across the device according to the Seebeck coefficient $\alpha$. The total heat pumped by the device can be found from the number $(\mathrm{N})$ of junctions (pairs of N-type and P-type semi-conductors), the ratio of the length to area of these junctions $(G)$ and the thermal conductance of the unit $(\kappa)$ for a given temperature distribution of the hot $\left(\mathrm{T}_{\mathrm{H}}\right)$ and cold $\left(\mathrm{T}_{\mathrm{C}}\right)$ faces, as a function of the supplied current [3];

$$
Q_{C}=2 N\left[I T_{C}-\frac{I^{2}}{2 G}-\left(T_{H}-T_{C}\right)\right]
$$

However, these equations can be simplified to obtain the linear resistance Seebeck co-efficient and conductance. These equations can be written after Luo and Bons as

$$
\begin{aligned}
& Q_{C}=-\kappa T_{H}+(\alpha I+\kappa) T_{C}-\frac{I^{2} R}{2} \\
& Q_{H}=\kappa T_{C}-(\alpha I-\kappa) T_{H}+\frac{I^{2} R}{2} \\
& V=\alpha\left(T_{H}-T_{C}\right)+I R
\end{aligned}
$$

The resistance of the unit is available from manufacturer data (validated by manufacturer measurements). This helps derive the Seebeck co-efficient and the conductance.

$$
\begin{gathered}
\alpha=\sqrt{\frac{2 R Q_{\max }}{T_{H}^{2}}} \\
\kappa=\frac{\alpha^{2}\left(T_{H}-\Delta T_{\max }\right)^{2}}{2 R \Delta T_{\text {max }}}
\end{gathered}
$$

Coefficient of performance $(Z)$ and figure of merit $(z T)$ are also very useful in determining performance of thermoelectric devices [4].

\subsection{Computational Tool}

The values defined above can be used to obtain the Seeback coefficient, conductance and resistance as a function of temperature. The values of $\alpha, \kappa$ and $R$ were used in a commercially available CFD package Star $\mathrm{CCM}+$ to provide heat flux boundary conditions for the thermoelectric cooling model (TEC). Two test modules were considered in the analysis, one Bismuth Telluride and the other custom hybrid bi-Te doped with lead. The linear equations were derived from the values obtained from manufacturer data sheets.

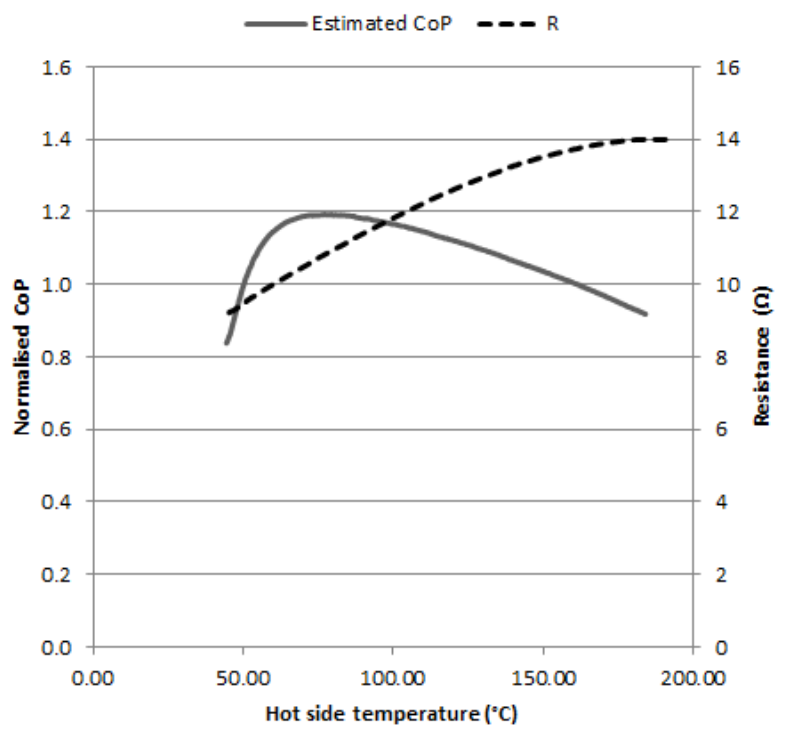

Figure 1: Experimental measurement of the resistance of the hybrid TEC unit

However, the model is valid in the tested temperature range but complications will arise at elevated temperatures as the thermal conductance, electrical resistivity and Seebeck coefficient for the thermoelectric modules vary with temperature, each material having differing characteristics. In order to derive the linear equations that define the performance of the TECs at elevated temperature it is necessary to re-evaluate the data sheet values for these temperatures. In the case of the Laird module, it is possible to use the manufacturers analytical design tool, Aztec (Scillasoft, 2014) to find these values at the system temperature. When such data are not available, an alternative approach has to be followed. The resistance of the unit at a range of mean temperatures was measured in a laboratory oven and a digital multi meter to record to voltage drop across the unit at a fixed current. From these data, a linear equation for the resistance could be derived. In the absence of direct experimental data and extrapolation technique based on measurements and curve fitting was used to find values of $z T$.

LaLonde et al. [6] plots values for $z T$ at temperature for a range of materials. If the composition of the module was known, values could be estimated from these curves. In the absence of these values, the data from the experimental oven testing was used to approximate $z T$ with temperature. A copper heat sink was used to dissipate heat from the hot side, and the cold side was fixed to an insulated mass. Thermocouples were used to measure the hot and cold side temperatures. An estimate of the cooling and the input power were used to find $z$ and the result normalised relative 
to the performance seen at $T_{H}=50{ }^{\circ} \mathrm{C}$, the data sheet value. This scale was then used to estimate $Q_{\max }$ and $\Delta T_{\max }$ and used to compute revised coefficients for the linear equations used in the model, using the methodology outlined above.

\subsection{Computational model}

The model is a three dimensional representation of the downhole tool manufactured by Avalon Sciences Ltd. It comprises a steel pressure barrel which houses the geophones, a mechanism to operate an arm which clamps the tool to the wall of the borehole and a module containing the digital electronics which perform the signal processing function. These electronics are housed within a vacuum insulated vessel and active cooling is provided by a Thermoelectric Cooler (TEC) module.

The model takes advantage of the symmetry of the tool; a representation of one half of the system is modelled, cut down the central axis of symmetry. Planar symmetry conditions are applied to the cut faces. The external region of the model, representing the well fluid, has a fixed temperature boundary condition on the far face, representing the large thermal capacity of the borehole fluid. The fluid is modelled in the laminar regime, with convection driven by gravity in the direction that the tool is oriented in the well. The well fluid is modelled as water. To fully resolve all of the electronic components housed within the module would incur a high computational cost to accurately resolve the geometry and thus a simplified representation of the PCB is used. The solid regions of the tool were modelled with appropriate material properties, sourced from the manufacturer's data sheet. The vacuum region of the flask is modelled as a gas with a low conductivity $\left(1 \times 10-{ }^{6} \mathrm{Wm}^{-1} \mathrm{~K}^{-1}\right)$. Surface to surface radiation is modelled, with the air in the spaces using the participating media model. Model initialized at borehole temperature would result in a converged solution at 4000 iterations.

\section{Results and validation}

A pumped hot oil bath was used to emulate the well fluid at elevated temperature and pressure. It is intended to take measurements in a well, but the controlled conditions in the laboratory represent the conditions down the hole. The limit of the fluid in the open system was $160{ }^{\circ} \mathrm{C}$. The tool was submersed in the fluid which was then heated. Once the system has reached steady state the temperature, as reported by a sensor embedded within the on-board electronics, was recorded. With the data from the experimental results at $160{ }^{\circ} \mathrm{C}$ external temperature the model was validated. The temperatures seen in the model are equivalent to those seen in the experimental testing. The model was then replicated with the derived parameters for the hybrid module, and the temperature field computed.

The main aim of the tool thermally is to isolate the hot fluid from the electronic circuitry and pump the heat generated out of the flask. Table 1 shows the fluid temperatures and flask temperatures with the cooling effect at a temperature difference.
Table 1: Summary of mean temperatures and cooling observed in experimental testing and simulation

\begin{tabular}{|c|l|l|l|l|}
\hline & $\begin{array}{l}\text { Fluid } \\
\text { Temp. } \\
\left({ }^{\circ} \mathrm{C}\right)\end{array}$ & $\begin{array}{l}\text { Flask } \\
\text { Temp. } \\
\left({ }^{\circ} \mathrm{C}\right)\end{array}$ & $\begin{array}{l}\text { Cooling } \\
\left({ }^{\circ} \mathrm{C}\right)\end{array}$ & $\begin{array}{l}\text { Voltage } \\
(\mathrm{V})\end{array}$ \\
\hline $\begin{array}{c}\text { Experiment } \\
\text { HT2 }\end{array}$ & 161.06 & 134.82 & 26.20 & 12.5 \\
\hline CFD HT2 & 160.00 & 133.77 & 26.20 & 11.6 \\
\hline $\begin{array}{c}\text { Experiment } \\
\text { TESH 127 }\end{array}$ & 160.37 & 127.57 & 32.80 & 16.5 \\
\hline $\begin{array}{c}\text { CFD TESH } \\
127\end{array}$ & 160.00 & 126.40 & 33.60 & 20.67 \\
\hline
\end{tabular}

Figures 2 and 3 show the temperature profiles obtained with the computational simulation.

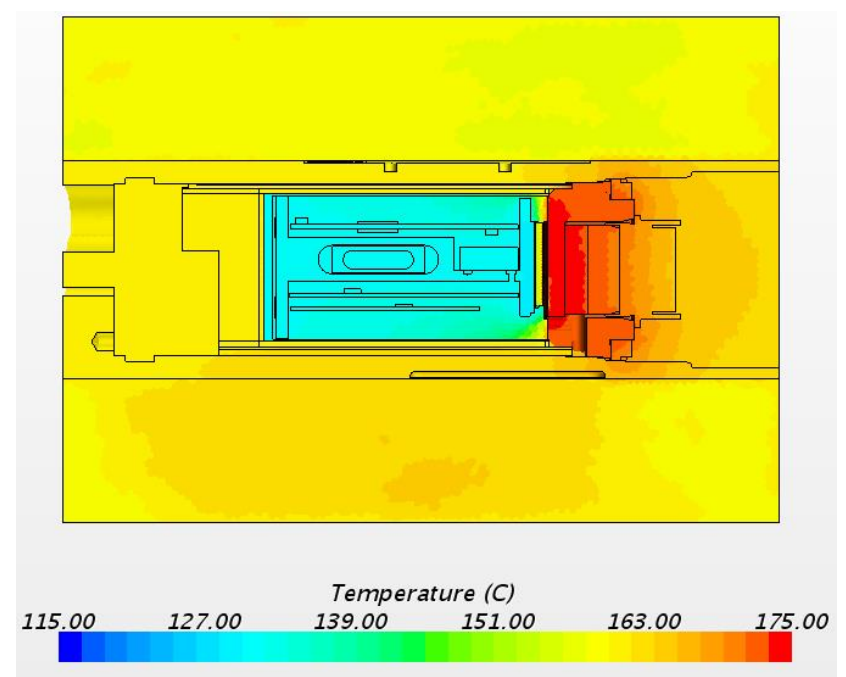

Figure 2: Temperature profile of the tool with the standard TEC module

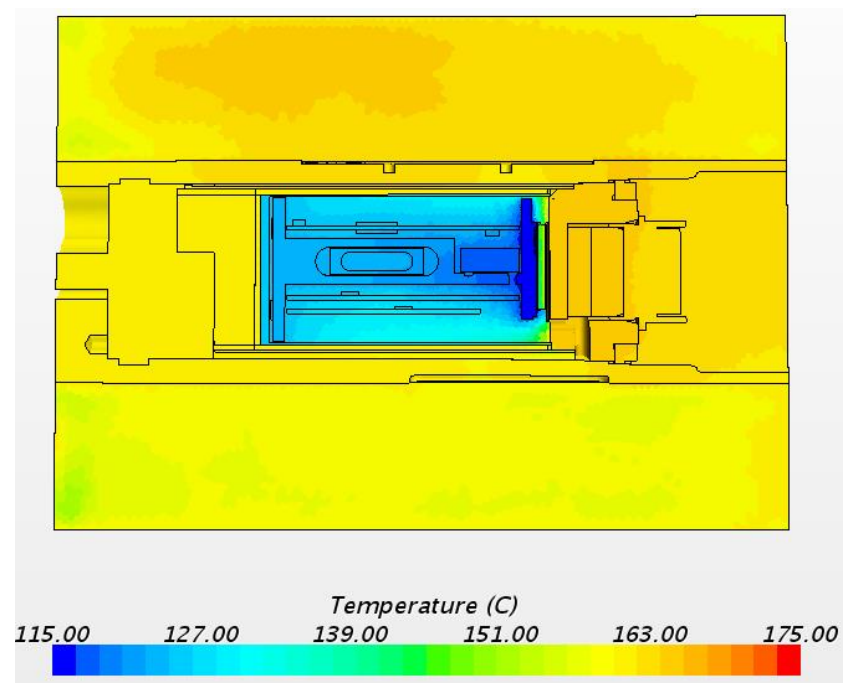

Figure 3: Temperature profile of the tool with the hybrid TEC module 


\section{Discussion and conclusions}

The main aim of the simulation work was to evaluate the performance of the downhole tool with two types of TEC cooling modules and then use the model as a base model in evaluating performance of similar tools. The numerical model was able to show the difference in performance of the two modules. The temperatures recorded in the electronic region of the module shows good agreement with the experimental values. However, the agreement on the voltage predictions is less impressive. This is, most likely due to the estimation of the $\alpha$ value.

The first order linear approximation method gives realistic values for the TEC properties at elevated temperatures. This is more true for $Q$ and $T$. It is yet to be confirmed how accurate it is for $\alpha$. A non-linear approximation method based on experimental values is under development at the moment. Initial investigations have shown that the relationship is better explained with second and third order terms. This should give better predictions at elevated temperatures. However with current capabilities and available knowledgebase, the numerical model has been able to predict the thermal behaviour of the downhole measuring tool. This has already shown to give huge benefits in design changes in tool geometry and TEC sizing. Further measurements, and with a more advanced prediction model, the numerical models are expected to give accurate models that will eliminate the use of testing, enabling the tool manufacturers to validate their designs without prior extensive testing.

\section{Acknowledgements}

The authors would like to thank Avalon Sciences Ltd for the continual support for the project given as the industry partner.

The research work was funded by the knowledge transfer partnership grant No KTP009448 of Innovate UK.

\section{Literature}

[1] G. Bennett, Active cooling for downhole instrumentation: design criteria and conceptual design summary. Office of Scientific and Technical Information, U.S. Dept. of Energy, [Online] available at: http://www.osti.gov/pages/servlets/purl/5507005, doi:10.2172/5507005. 1986.

[2] G. Bennett, G. Sherman, Analysis and thermal-design improvements of downhole tools for use in hot-dry wells. 1983 [Online] available at: http://www.osti.gov/pages/190 servlets/purl/6491119, doi:10.2172/6491119.

[3] Shengqiang Bai, Hongliang Lu, Ting $\mathrm{Wu}$, Xianglin Yin, Xun Shi, and Lidong Chen. Numerical and experimental analysis for exhaust heat exchangers in automobile thermoelectric generators. Case Studies in Thermal Engineering, 4: pp 99-112, 2014.

[4] Zhiting Tian, Sangyeop Lee, and Gang Chen. A comprehensive review of heat transfer in thermoelectric materials and devices. Ann. Rev. Heat Transfer, 17: pp.425-483, 2014.

[5] R. Bons, Efficiently simulating thermoelectric devices and heat pipes.[Online], available at: http://www.cdadapco.com/webinar/efficiently-simulatingthermoelectric-devices-and-heat-pipes.

[6] A.D. LaLonde, Y. Pei, H. Wang and G.J. Anyder Lead telluride alloy thermoelectrics. Materials Today 14, pp. $526-532,2011$ [Online], available at: http://www.sciencedirect.com/science/article/pii/S1369 702111702784,doi:http://dx.doi.org/10.1016/S13697021(11)70278-4. 